\title{
ANÁLISE EPIDEMIOLÓGICA DE 266 DECLARAÇÕES DE ÓBITO POR MALÁRIA REGISTRADAS EM GOIÁS, NO PERÍODO DE 1981 A 1993
}

Luciana Leite Pineli, ${ }^{1}$ Elizabeth da Silva Oliveira Araújo, ${ }^{2}$ Cecilio Alves de Moraes, ${ }^{3}$ e Joaquim Caetano de Almeida Netto ${ }^{4}$

\section{RESUMO}

Foram analisadas 266 declaraçð̃es de óbito de pacientes com causa de morte atribuida à malária, registradas no Estado de Goiás, no período de 1981 a 1993, no tocante à idade, sexo, profissão, residência, naturalidade, local do óbito e disfunçð̃es orgânicas responsabilizadas pelo evento. A análise ressalta que os óbitos por malária ocorreram mais em homens em idade acima de 20 anos que exerciam profissðes relacionadas a atividades extrativistas, portanto com nítido risco profissional. As disfunções orgânicas mais freqüentes, embora com diagnóstico nem sempre embasado em dados laboratoriais, foram a renal, seguida da neurológica, da cárdiorespiratória e da hepática. Correlacionando as médias anuais de óbitos por malária, de lâminas positivas, e da população no período do estudo, obtêm-se para o Estado de Goiás coeficientes de morbidade de 0,24 casos/1000 habitantes, de mortalidade de 0,005 obitos/1000 habitantes e de letalidade de 2,3 óbitos / 100 casos, no referido período.

UNITERMOS: Malária por P. falciparum. Morbimortalidade. Letalidade. Disfunções orgânicas.

\section{INTRODUÇÃO}

Apesar de a malária ser uma doença muito antiga e dos esforços desenvolvidos para seu controle $(6,7,9,11)$, ela continua ocupando um lugar de destaque a nível mundial, dentre as doenças transmissíveis. Segundo a Organização Mundial de Saúde (O.M.S.), mais de 2 bilhões de pessoas estão expostas a variados níveis de risco de contrair a doença em cerca de 100 países, estimando-se que ocorram anualmente mais de 200 milhões de casos e

1 Monitora do Departamento de Medicina Tropical - Instituto de Patologia Tropical e Saúde Pública (IPTSP) - Universidade Federal de Goiás (UFG)

2 Médica do Setor de Vigilância Epidemiológica da Fundação Nacional de Saúde em Goiás

3 Professor Auxiliar do Departamento de Saúde Coletiva / IPTSP / UFG

4 Professor Titular do Departamento de Medicina Tropical / IPTSP / UFG

Endereço para correspondência: Joaquim C. Almeida Netto, IPTSP, UFG, Caixa Postal 131, CEP: 74001-170, Setor Universitário. Goiânia, GO.

Recebido para publicação em 18/03/97. Revisto em 09/09/97. Aceito em 13/11/97. 
cerca de 1,5 milhão de óbitos, sendo, nas regiões endêmicas, as crianças as principais vítimas $(10,11,14)$.

A grande maioria dos casos ocorre em países tropicais da África Ásia e América Latina. No Brasil, a malária está praticamente confinada à Região Amazônica que concentra mais de $95 \%$ dos casos (9). Quanto à morbi-mortalidade e, principalmente, à letalidade, a maioria dos dados disponíveis refere-se a casuísticas de hospitais de referência não retratando a realidade da doença, pois os casos hospitalizados são os que apresentam sintomas clínicos mais acentuados e, portanto, os mais graves, constituindose assim numa amostra selecionada.

No Estado de Goiás, numa série de 1.379 pacientes de malária por $P$. falciparum internados no período de 1983 a 1994 no Hospital de Doenças Tropicais de Goiânia (HDT-GO), referência regional para doenças transmissíveis, $15,5 \%$ eram portadores da forma grave e complicada da doença e 4,5\% evoluíram para óbito (1). Já os dados oficiais apontam uma letalidade média de $1,1 \%$ no período de 1984 a 1994 para o Estado de Goiás e de $2,8 \%$ para o Distrito Federal (5).

O presente trabalho tem como objetivo analisar as características epidemiológicas associadas aos óbitos por malária registrados no Estado de Goiás, buscando conhecer, nesta doença, os fatores intervenientes e as disfunções orgânicas mais assinaladas como responsáveis pela sua evolução fatal. Pretende ainda construir para o Estado de Goiás uma estimativa dos coeficientes de morbidade, mortalidade e letalidade com base no número de óbitos notificados, no número de lâminas positivas e nos dados disponíveis sobre a população.

\section{MATERIAL E MÉTODOS}

Foram analisadas 266 declarações de óbitos, cuja causa básica foi referida como "malária", "malária falciparum" e "malária mista", registradas na Secretaria de Ações Básicas de Saúde do Estado de Goiás (SABS/GO) no período de 1981 a 1993. Foram levantados os dados referentes a sexo, idade, residência, atividade profissional, local de atendimento e do óbito e as disfunções orgânicas assinaladas como causas do evento.

Os dados em estudo foram lançados em ficha própria para entrada em microcomputador e para análise dos dados epidemiológicos levantados. A média anual de óbitos foi analisada em relação à média anual da população e de lâminas positivas (Cenepe, 1993) (4), e destas com a média populacional (IBGE, 1994) (8).

\section{RESULTADOS}

Em Goiás, no período de estudo, foram notificados pela SABS/GO 266 óbitos por malária, observando-se um nítido decréscimo do número anual de óbitos a partir de 1989 e de lâminas positivas, a partir de 1991, (Tabela 1).

Tabela 1. Estimativa de Morbidade, Mortalidade e Letalidade da malária em Goiás - 1981 a 1993

\begin{tabular}{c|c|c|c|c|c|c}
\hline Ano & $\begin{array}{c}\text { Obitos } \\
\text { por } \\
\text { malária }\end{array}$ & $\begin{array}{c}\text { Lâminas } \\
\text { positivas }\end{array}$ & $\begin{array}{c}\text { População de } \\
\text { Goiás }\end{array}$ & $\begin{array}{c}\text { Morbidade } \\
\text { (X1.000) }\end{array}$ & $\begin{array}{c}\text { Mortalidade } \\
\text { (X1.000) }\end{array}$ & $\begin{array}{c}\text { Letalidade } \\
\text { (X100) }\end{array}$ \\
\hline 1981 & 40 & 712 & 3.192 .100 & 0,22 & 0,012 & 5,62 \\
1982 & 47 & 742 & 3.278 .500 & 0,22 & 0,014 & 6,33 \\
1983 & 37 & 1502 & 3.365 .300 & 0,44 & 0,007 & 2,46 \\
1984 & 24 & 1074 & 3.452 .500 & 0,31 & 0,007 & 2,23 \\
1985 & 22 & 822 & 3.539 .900 & 0,23 & 0,006 & 2,68 \\
1986 & 11 & 1160 & 3.626 .300 & 0,32 & 0,003 & 0,95 \\
1987 & 15 & 1347 & 3.710 .800 & 0,36 & 0,004 & 1,11 \\
1988 & 26 & 980 & 3.793 .200 & 0,26 & 0,007 & 2,65 \\
1989 & 10 & 1191 & 3.873 .500 & 0,31 & 0,003 & 0,84 \\
1990 & 11 & 746 & 3.951 .400 & 0,19 & 0,003 & 1,47 \\
1991 & 10 & 507 & 4.027 .000 & 0,13 & 0,002 & 1,97 \\
1992 & 7 & 376 & 4.100 .400 & 0,07 & 0,002 & 1,86 \\
1993 & 6 & 244 & 4.171 .500 & 0,06 & 0,001 & 2,46 \\
\hline Média & & & & & & \\
anual & 20,46 & 877,15 & 3.698 .646 & 0,24 & 0,005 & 2,33 \\
\hline
\end{tabular}

A análise das 266 declarações de óbito, segundo os dados epidemiológicos pesquisados, está apresentada nas tabelas 2 e 3 , e nas figuras 1 e 2 . Ressalta-se que, dentre os 266 casos fatais, a maioria constituía-se de homens $(71,8 \%)$, com idade acima de 20 anos $(67,7 \%)$, naturais dos Estados de Goiás e Tocantins $(85,8 \%)$, exercendo atividades extrativistas ou a elas relacionadas $(71,4 \%)$. A maioria dos óbitos $(65 \%)$ ocorreu no interior dos Estados de Goiás e Tocantins, com as seguintes disfunções relacionadas ao evento: em 68 casos $(23,4 \%)$, renal; em 65 (22,2\%), neurológica; em 58 $(19,8 \%)$, cárdio-respiratória; em $48(16,4 \%)$, hepática; em $30(10,3 \%)$, hematológica; em $17(5,8 \%)$, metabólica e $6(2,1 \%)$, falência de múltiplos órgãos.

Ressalta que a disfunção cerebral foi a mais freqüente em crianças e a renal nos adultos (figura 2). 
Tabela 2. Dados epidemiológicos em 266 óbitos por malária no Estado de Goiás, 1981 a 1993.

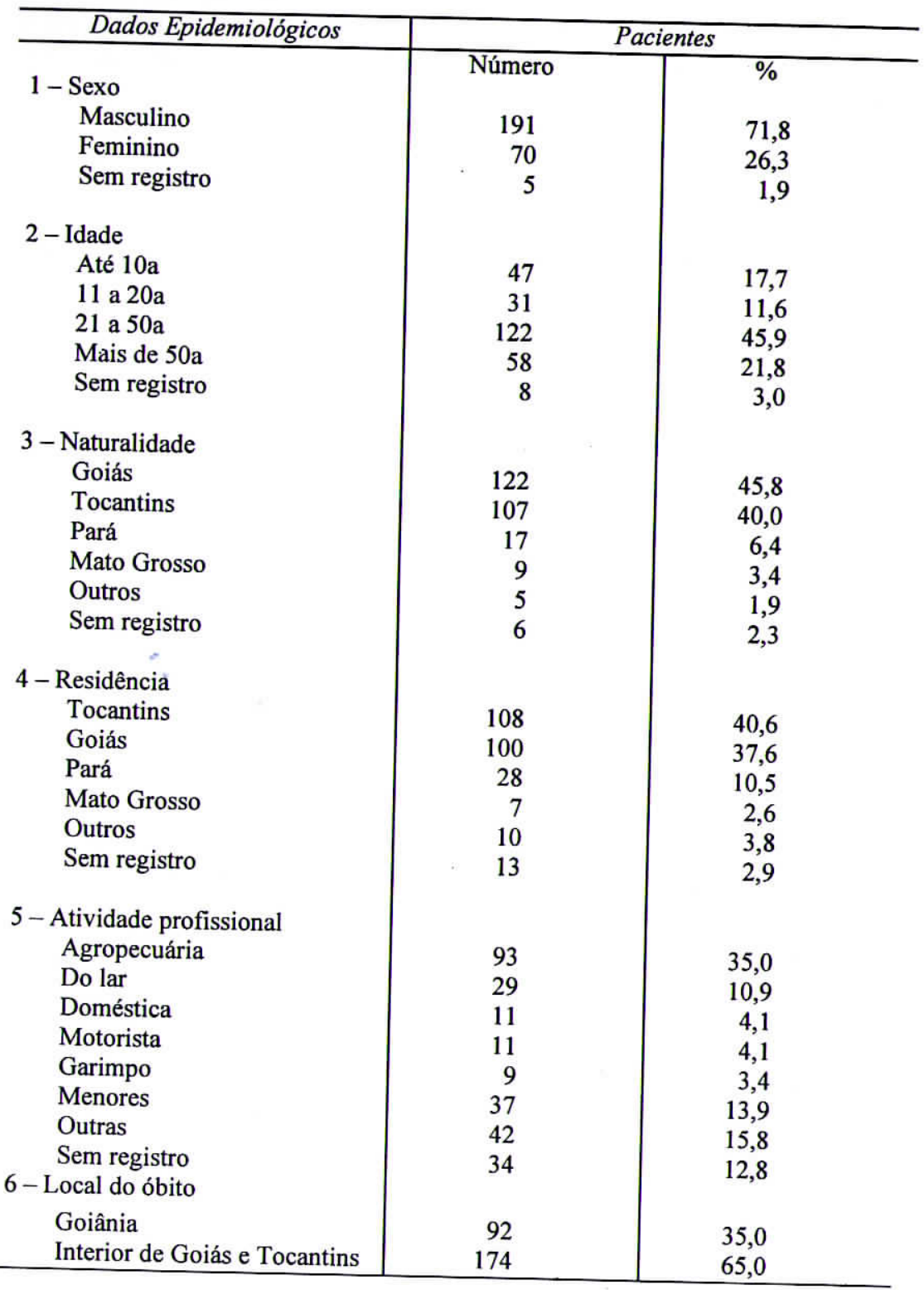

Tabela 3. Disfunçð̃es orgânicas responsabilizadas pela morte em 266 atestados de óbito por malária no Estado de Goiás, 1981 a 1993.

\begin{tabular}{|c|c|c|}
\hline Disfunções orgânicas & $\mathrm{N}^{\circ}$ & $\%$ \\
\hline Renal & 68 & 23,4 \\
\hline Neurológica & 65 & 22,2 \\
\hline Cárdio-respiratória & 58 & 19,8 \\
\hline Hepática & 48 & 16,4 \\
\hline Hematológica & 30 & 10,3 \\
\hline Metabólica & 17 & 5,8 \\
\hline Falência múltipla & 6 & 2,1 \\
\hline Total & $292^{*}$ & 100,0 \\
\hline
\end{tabular}

\section{DISCUSSÃO}

Os dados da série em estudo mostram que houve um nítido declínio no número de óbitos a partir de 1989 (Tabela 1), coincidentemente com a criação do Estado do Tocantins e com a diminuição das atividades de garimpo na Região Amazônica. Por outro lado, a maioria dos óbitos por malária incidiu em indivíduos do sexo masculino na faixa etária de 20 a 50 anos (Tabela 2), fato certamente relacionado às migrações internas no país, predominantemente de indivíduos adultos do sexo masculino em busca de trabalho, principalmente na Região Amazônica $(7,9)$.

Comparando-se a distribuição dos óbitos por malária nas crianças e adolescentes, adultos e idosos com a composição da população do Estado de Goiás (8), segundo as mesmas faixas etárias (Figura 1), observa-se que não houve paralelismo entre estes dados, o que demonstra haver uma diferença na exposição à doença nos grupos etários estudados, situação que demonstra seu caráter profissional na região do estudo, uma vez que a malária acomete mais homens adultos que migram para áreas malarígenas em busca de trabalho, relacionado, na maioria das vezes, às atividades extrativistas $(7,9)$.

Dos 1.379 pacientes de malária internados no Hospital de Doenças Tropicais (HDT-GO), 39,1\% se ocupavam com atividades de garimpo e $31,9 \%$ com atividades agropecuárias (1). Constatou-se, porém, que a maioria dos óbitos ocorreu entre os trabalhadores rurais (1). Este fato pode ser explicado pelo conhecimento que os garimpeiros, pela sua freqüente condição de reinfectado, têm da doença. Submetendo-se à hemoscopia logo no início da sintomatologia e realizando profilaxia com antimaláricos, os garimpeiros se cercam de condições que podem protegê-los contra o óbito (1,
$7,10)$.

Observa-se ainda (Tabela 2) que a maioria dos óbitos ocorreu em pacientes residentes nos Estados de Tocantins e Goiás e em número significativamente menor nos Estados do Pará e Mato Grosso. Todavia, na 
série de pacientes estudada em Goiânia (1), a maioria dos doentes embora residentes em Goiás e no Tocantins, procediam dos Estados do Mato Grosso (40\%) e do Pará (39\%) mostrando nessa série a grande importância das migrações internas na exposição à doença $(7,9,11)$. Infelizmente, no presente estudo, a procedência dos casos fatais de malária não pode ser avaliada, pelo fato de este dado não constar do formulário para registro de óbitos em uso no Brasil.

Dentre as disfunções orgânicas responsabilizadas pelo óbito (Tabela 3) a mais freqüente foi a renal, seguida da neurológica e da cárdiorespiratória. Nas crianças predominou a disfunção cerebral, nos adultos a renal e nos idosos a cárdio-respiratória. Já nos adolescentes a ocorrência das disfunções foi homogênea (Figura 2).

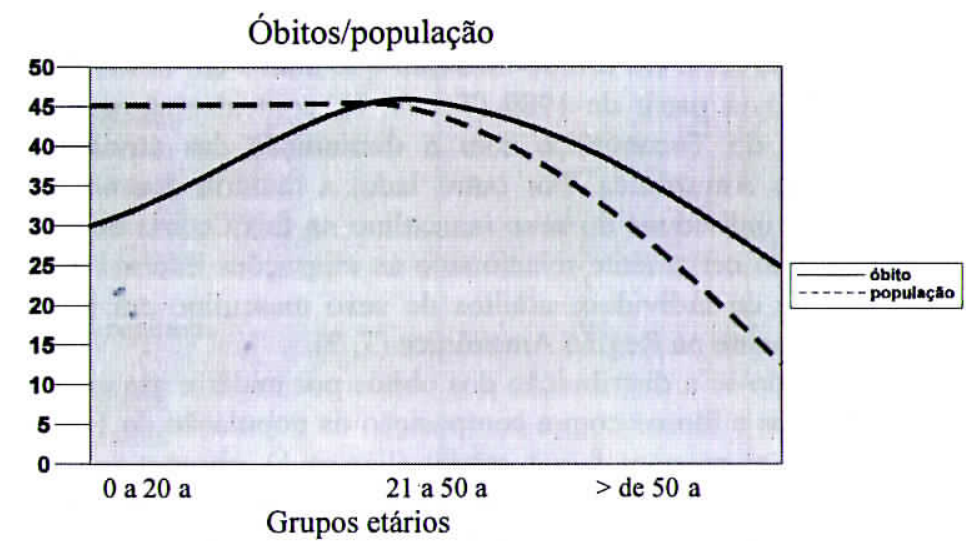

Figura 1. Distribuição percentual de 258 óbitos por malária e da população do Estado de Goiás, segundo grupos etários, 1981 a 1993.
田Neurológica —Cárdio-resp. DRenal DHepática

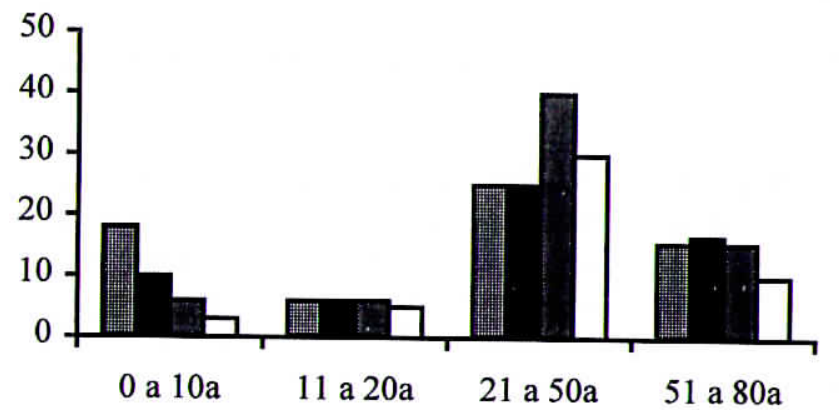

Figura 2. Óbitos por malária - Distribuição das quatro principais disfunções orgânicas, segundo grupos etários em 258 declarações de óbito em Goiás, 1981 a 1993.

Nas regiões endêmicas as formas graves da doença e, conseqüentemente, os óbitos incidem mais nas crianças, sendo a disfunção orgânica mais freqüente a cerebral $(10,11,14)$. Como, na série de óbitos em estudo, a maioria dos pacientes era constituída de adultos, a maior prevalência da disfunção renal está em consonância com os dados da literatura $(2,3,10,13,14)$. Na série de 1.379 pacientes de malária por $P$. falciparum estudados em Goiânia (2), bem como na série de 3.985 pacientes estudados em Manaus (3), ambas, em sua maioria constituídas por indivíduos adultos, a complicação mais importante foi a renal.

Por outro lado, considerando-se, no período de estudo, a média anual da população (8), o total de lâminas positivas (4) e de óbitos, com valores respectivos de 3.698.646 habitantes, 877 lâminas positivas e 266 óbitos, pode-se projetar para o Estado de Goiás coeficientes médios de morbidade, mortalidade e letalidade de 0,24 casos/ 1.000 habitantes, 0,005 óbitos/1.000 habitantes e 2,33 óbitos/100 casos, respectivamente.

Comparando-se o número de óbitos por malária com os provocados por outras doenças, ocorridos no HDT-GO, referência regional para doenças transmissíveis, verifica-se que a malária foi responsável por 63 óbitos de um total de 1.088 ocorridos no período do estudo (12), o que corresponde a um coeficiente de mortalidade proporcional de $6 \%$. Assim, a malária ainda é uma importante causa de morte em uma região considerada como de baixo risco de transmissão (9). Esse dado certamente deve ser creditado à alta ocorrência 
de casos importados, da Região Amazônica, com alto risco de desenvolver a forma grave da doença.

\section{SUMMARY}

Epidemiologic analysis of 266 deaths due to malaria in Goiás state from 1981 to 1993

Death certificates of 266 patients who had died from malaria in Goias State from 1981 to 1993 were analyzed, in regard to age, gender, place of birth, place of death and other related pathologies accountable for the lethal outcome. Mortality was higher on 20 year old age group, all males that worked on the fields or gold mines, reflecting the risk of this occupations. The death by malaria more frequently occurred in men over 20 years, that exerted agricultural and gold miner activity, so in workers under professional risk conditions. Associated diseases were mainly renal, neurologic, cardiac, respiratory and hepatic. We obtained the following coefficients after correlating the annual average of deaths by malaria, positive slides and population during the studied period: morbility ratio of 0,24 cases $/ 1000$ inhabitants, mortality ratio of 0,005 deaths $/ 1000$ inhabitants and case fatality of 2,3 deaths $/ 100$ cases.

KEYWORDS: Plasmodium falciparum. Malária. Morbidity. Mortality. Case fatality. Organic disfunctions.

\section{REFERÊNCIAS BIBLIOGRÁFICAS}

01. Almeida Netto, J.C. Perfil epidemiológico e letalidade associada em pacientes com malária por $P$. falciparum internados em Hospital de Referência de área não endêmica. Rev. Soc. Bras. Med. Trop., (Supl.1), 28, 106, 1995.

02. Almeida Netto, J.C.; Natal, M. E. L.; Santos, E.R. e Pereira, L. I. A. Disfunçðes orgânicas e letalidade associada em pacientes com malária por $P$. falciparum internados em Hospital de refêt.): 16, 1995

03. Bubol, W.S.; Siva E. B.; Farias, F. E. S.; Alecrim, M. G.; Sampaio,M.Z.M.; Alecrim, W. D. Insuficiência renal aguda em malária falciparum. Experiência em 159 casos. Anais do XXVII Congresso da Sociedade Brasileira de Medicina Tropical, Belém, Vol. II, p. 9, 1992.

04. Centro Nacional de Epidemiologia (Cenepi) - Ministério da Saúde - Notificação de lâminas positivas para $P$. falciparum, 1994.

05. Codepro/Fundação Nacional da Saúde. Internações, óbitos e taxas de letalidade em hospitais, de 1984 a 1994. Programa de Controle da Malária na Bacia Amazônica. P. 1 e 2. Brasilia, 1994.

06. Deane, L.M. Os grandes marcos na história do controle da malária. Rev. Soc. Bras. Med. Trop. 25 (Supl.II): 12-22, 1992.

07. Fundação Nacional da Saúde / MS. Controle da malária. Diretrizes técnicas., Brasília, 1995. 48p.

08. Instituto Brasileiro de Geografia e Estatística. Anuário estatistico do Brasil. p. 2-14. 1994
09. Marques, A. C. Gutierrez, H. C. Combate à malária no Brasil: Evolução, situação atual e perspectiva. Rev. Soc. Bras. Med. Trop. 27 (Supl. II): 91-108, 1994

10. Organização Mundial de Saúde. Tratamento da malária grave e complicada - condutas práticas, 56 p., Brasilia, 1995.

11. Oficina Sanitária Panamericana. La lucha mundial contra el paludismo. Bol. Ofic. Sanit. Panam. 116: 477-482, 1994

12. SAME. Relatório anual - Hospital de Doenças Tropicais. 1981 - 1993.

13. Trang, T. T. M., Phu, N. H., Vinh, H., Hien, T. T., Cuong, B. M., Chau T. T. H., Mai, N. T. H., Waller, D. J., White, N. J. Acute renal failure in patients with severe falciparum malaria. Clin. Infect. Dis., 15:84-80, 1992.

14. Warrel, D. A. Molineaux and P. F. Beales. Severe and complicated malaria. Trans. Roy. Soc. Trop. Med. Hyg, 84 (Supl 2): 1-65, 1990. 